\title{
La participación de mujeres y hombres en los directorios de las empresas más exitosas de Estados Unidos: ¿tiene alguna relación con el rendimiento de la compañía?
}

\section{Bárbara Reyes Molina, Alex Medina Giacommozi}

\author{
Universidad del Bío-Bío, Chile \\ Facultad de Ciencias Empresariales
}

No existen estudios claros que expliquen si existe una relación entre el sexo de los directores y su rendimiento; sin embargo, muchos estudios señalan ventajas de contar con participación femenina dentro de los consejos de administración. Catalyst (1995) indica que, al aumentar la diversidad, incrementa la pluralidad en las opiniones del directorio. Por su parte, Bilimoria (2006) expresa que el género femenino entrega mejores estrategias y asegura un mejor comportamiento en las reuniones. A su vez, Kuczynski (1999) afirma que un directorio más diverso puede enfrentar de mejor manera los problemas entre miembros y genera mejores decisiones, por lo que una compañía es vista como menos riesgosa; por ello, una directora debería mejorar el desempeño de la compañía.

A partir de ello, este estudio pretende identificar la participación de la mujer en los directorios de una muestra de 60 empresas del ranking Fortune 500 año 2014 (Fortune, 2014), con el fin de establecer una relación entre la diversidad de sexo en los directorios y el rendimiento de una empresa, mediante una regresión cualitativa Logit.

La participación de la mujer en los directorios de compañías estadounidenses ha aumentado; no obstante, sigue siendo débil en comparación con la de los hombres: en promedio, el directorio está compuesto por once miembros, de los cuales, aproximadamente, nueve son hombres y dos, mujeres. Hay compañías que no incluyen mujeres en las juntas. En esta investigación, se concluye que el sexo no es una variable estadísticamente significativa que interviene en el rendimiento de una organización.

Palabras clave: sexo, rendimiento, directorio, gobiernos corporativos, Logit.

\section{Men and women as board directors of the most successful companies in the USA: Is there any connection with organizational performance?}

There are no clear studies that explain whether there is a relationship, but many authors point out advantages of having female participation in boards of directors; Catalyst (1995) states that increasing diversity, there is a plurality of opinions in the boardroom; and Bilimoria (2006) states that women deliver the best strategies and ensure better behavior at meetings. Likewise, Kuczynski (1999), said that a more diverse boardroom can handle problems between members better and it can lead to better decisions, so it is seen as a less risky company, therefore, it is expected that the presence of women improve organizational performance.

This study aims to identify the participation of women on the boards of directors of 60 companies in Fortune 500 in 2014 (Fortune, 2014). A qualitative Logit regression was applied in order to establish a relationship between gender diversity in directive boards and the company's performance. 
The participation of women in American companies has increased; however, numbers remain low compared to men because, on average a board of directors has 11 members, and on average 9 are men and 2 are women. There are companies that do not include women in the boardroom. In this research, we conclude that gender is not a significant statistical variable involved in the performance of a company.

Keywords: gender, performance, board of directors, corporate governance, Logit.

\section{A participaçáo das mulheres e dos homens nos diretórios das empresas mais bem sucedidas nos EUA: existe alguma conexáo com o desempenho da empresa?}

Não há estudos claros que explicam a existência de uma relação entre o gênero dos diretores e o desempenho empresarial. No entanto, muitos estudos apontam vantagens de ter participaçáo feminina na diretoria. Catalyst (1995) indica que a diversidade aumenta a pluralidade de opinióes. Enquanto isso, Bilimoria (2006) afirma que as mulheres proporcionam melhores estratégias e garantem um melhor comportamento nas reuniōes. Por sua vez, Kuczynski (1999) afirma que uma diretoria mais diversificada pode enfrentar melhor os problemas entre os membros e gerar melhores decisões, de modo que uma empresa é vista como menos arriscada; portanto, uma diretora deve melhorar o desempenho da empresa.

Este estudo tem como objetivo identificar a participação das mulheres na diretoria de 60 empresas da Fortune 500, 2014 (Fortune, 2014), a fim de estabelecer uma relaçáo entre a diversidade de gênero na diretoria e o desempenho de uma empresa através da regressão Logit qualitativa.

A participação das mulheres na direção de empresas norte-americanas aumentou; no entanto, continua baixa em comparação com a dos homens: em média, o conselho de administração é composto por 11 membros, dos quais 9 são homens e 2 mulheres. Ainda existem empresas que não incluem mulheres nos conselhos. Nesta pesquisa, concluímos que o gênero não é uma variável estatisticamente significativa que afete o desempenho de uma organização.

Palavras-chave: gênero, desempenho, conselho de administração, governança corporativa, Logit.

\section{Introducción}

La diversidad se puede clasificar en distintas dimensiones, como el sexo, la raza o la etnia. La diversidad en cuanto al sexo no es una característica de las juntas directivas alrededor del mundo; en otras palabras, la inclusión de la mujer en los cargos directivos de una compañía es mucho menor que la de hombres. En el mundo, la incorporación de la mujer en las juntas directivas ha sido un proceso lento, pero ha aumentado a través de los años (Jara, 2014). Bilimoria y Piderit (1994) estudiaron 300 empresas del listado Fortune, en que se identificó que la participación masculina era muy superior a la femenina. Wheelen, Hunger y Oliva (2007) entregan algunos datos respecto a la evolución de este tema: Korn/Ferry International informó en un estudio que el $80 \%$ de las empresas estadounidenses del ranking Fortune 1000 tenía para el año 2003 al menos una directora dentro de la junta, en comparación con el $74 \%$ en el año 2000, lo que representa un $13,6 \%$ de todos los directores estadounidenses. En Estados Unidos, para el año 2007, de acuerdo con un estudio realizado a empresas pertenecientes al listado Fortune 500, se determinó que la participación de la mujer en los directorios era de un 14,87\% (Johnson, Scholes \& Whittington, 2006). 
Por su parte, Oakley (2000) indica que la participación de la mujer en los altos cargos directivos ha crecido rápidamente en las últimas dos décadas, pero sigue siendo bajo en comparación a los hombres, debido a la falta de experiencia en línea, oportunidades de carrera, diferencias de sexo en los estilos de sociabilización y comunicación, paradigmas de género y la edad. En otro estudio sobre este tema, Burgess y Tharenou (2002) reconocen la baja participación de la mujer e identifican que una de las causas de esto es la cultura tradicional, en la que la participación del hombre es predominante. Desde otro ángulo, Williams (2003), quien investigó 185 empresas del listado Fortune 500 entre 1991-1994, señala que existe una relación negativa entre la cantidad de mujeres en el directorio y la filantropía. Mientras, Adams y Ferreira (2004) reconocen que existe baja participación femenina y resumen su investigación en tres puntos centrales: las empresas que tienen mayor volatilidad en el mercado de acciones tienen menor cantidad de mujeres en sus juntas directivas; aquellas compañías que poseen mayor diversidad en el consejo ofrecen mejores salarios a sus participantes; $y$, finalmente, afirman que, a mayor diversidad, aumenta el número de reuniones debido a que las mujeres rebaten las decisiones (Adams \& Ferreira 2004). Unos años después, los mismos autores señalaron que debería aumentar la participación femenina, debido a las presiones ejercidas a empresas para integrar a la mujer dentro de los miembros de la junta directiva (Adams \& Ferreira, 2008).

Sin embargo, no existe un estudio claro que determine si existe una relación positiva o negativa entre la diversidad de género y el desempeño de la empresa. Adams y Ferreira (2008) estudiaron si existía una variación entre asistencia y asignación de comités con la diversidad, si la forma en que se componía el directorio afectaba las medidas de gobernabilidad como las adquisiciones y la compensación de los CEO y si la diversidad podía afectar el desempeño de la firma.
En conclusión, se determinaba que las mujeres son distintas en comportamiento a los hombres, por lo que aparentemente parecían tener menos problemas de asistencia y, a su vez, probablemente participaban más en los comités de monitoreo que los hombres. Por ello, es más probable encontrar a una mujer en auditoría que en un comité de compensación. En cuanto a las adquisiciones y compensación de los CEO, existe mayor probabilidad de que, en aquellos directorios con mayor diversidad, el movimiento del CEO sea más sensible al desempeño del precio de la acción. No se encontró una relación estadística entre diversidad y compensación, pero estos autores determinan que las juntas más diversas reciben compensaciones más equitativas. El último punto de estudio, la relación entre diversidad y desempeño de la empresa, no es claro, ya que, en primera instancia, pareciera ser una relación positiva; no obstante, a medida que se incluyen otras variables y problemas de causalidad, se desvanece la teoría (Adams \& Ferreira, 2008). Torchia, Calabro y Huse (2011) señalan que la participación femenina representa una pequeña minoría en las juntas directivas a pesar de que tiene una importancia estratégica, debido a que, al aumentar la participación de mujeres en el directorio, mejora los niveles de innovación de la empresa. Ernst \& Young (2013) determinan que existe un progreso en las juntas directivas en Estados Unidos, donde la mujer se ha incorporado a tasas que incrementan con los años y que existe mayor probabilidad de que una empresa que ya tiene mujeres incorpore a otra que una empresa que no tiene dentro de sus miembros de consejo a una mujer. En esa línea, Tacheva y Huse (2006), luego de un análisis de 249 empresa noruegas determinó que la presencia de mujeres en el directorio contribuía a la eficiencia y dinámica de la junta. Sin embargo, Caprino (2013) resalta que se ha progresado muy poco en el cambio corporativo en cuanto a equilibrio de género. 
Atribuye esto a que las empresas no están lo suficientemente preocupadas sobre esta situación (Caprino, 2013), pese a que, como explican Carter, Simkins y Simpson (2002) luego de una investigación del listado Fortune 1000, existe una relación positiva entre diversidad y valor de la empresa. Este resultado es respaldado por Erhardt, Werbel y Shrader (2003), quienes estudiaron la diversidad de 127 empresas muy importantes de Estados Unidos e identificaron que a diversidad del directorio tiene una relación positiva con el desempeño financiero.

Desde hace muchos años, existe la preocupación por la desigualdad de género en los directorios de las empresas alrededor del mundo. Todos los autores anteriormente citados reconocen que la participación de la mujer en las juntas directivas ha crecido con los años, pero sigue siendo muy baja. Además, expresan que la participación femenina trae diversas ventajas para las organizaciones que incluyen directoras en la junta de administración. El objetivo de esta investigación es analizar la participación de la mujer en los directorios de las empresas con mayor volumen de ventas de Estados Unidos de acuerdo con el listado Fortune 500 del año 2014 (Fortune, 2014) y establecer la posible relación entre sexo de los directores y rendimiento de la empresa.

\section{Metodología de la investigación}

Los datos recolectados en esta investigación son de tipo cualitativo y cuantitativo, por lo que, de acuerdo a este criterio, la investigación sigue un carácter mixto. Esta se ha basado en un estudio observacional; es decir, no se pretende controlar el resultado de las variables, sino analizar cada una de estas y establecer si existe una relación entre ellas. Asimismo, cabe anotar que, de acuerdo a la relación cronológica, el estudio es retrospectivo.
La población para este estudio corresponde a las 500 empresas incluidas en el listado Fortune 500 del año 2014 (Fortune, 2014). Este ranking incorpora, año tras año, a las compañías estadounidenses con mayores niveles de ventas a nivel global, tanto dentro como fuera del país. El ranking es publicado por la revista Fortune, y en este se incluyen organizaciones públicas y privadas, que coticen en bolsa, incluidos los rubros de minería, servicios, manufactura, financieros, energía, entre otros. De acuerdo a una población de 500 empresas estadounidenses publicadas en el Listado Fortune 500 del año 2014, se estableció una muestra de 60 compañías, con un $90 \%$ de confianza. La selección de las empresas fue a través de un sistema de muestro aleatorio simple.

Luego de obtener las empresas, se identificó el nombre de los directores, el sexo y el rendimiento de la compañía, que, para los efectos de esta investigación, viene dado por el retorno sobre la inversión que publica el Ranking Fortune 500 en la web oficial. Una vez obtenido estos datos, se analizaron los datos a través de las principales medidas estadísticas para luego aplicar un modelo Logit.

\section{Resultados}

\subsection{Análisis variable rendimiento}

\subsubsection{Distribución de frecuencias}

El primer intervalo va desde un rendimiento de $3 \%$ a $24 \%$ y contiene a 22 empresas que representan un $37 \%$ de los datos de la muestra. El siguiente ítem contiene rendimientos desde un $24 \%$ a un $44 \%$ y tiene 17 compañías, que ponderan un $28 \%$ de los datos. El tercer intervalo va desde $44 \%$ a $64 \%$; forman parte de este 9 organizaciones, que acumulan el 15\% de las firmas de la muestra. La cuarta clase va desde $64 \%$ a $85 \%$ y está constituida por 10 empresas, que representan un $17 \%$ de los datos. Finalmente, está el quinto intervalo,

Contabilidad y Negocios (11) 22, 2016 / ISSN 1992-1896 
que va desde $85 \%$ a un $105 \%$ y tiene 2 empresas que acumulan un $3 \%$ de la muestra. Cabe anotar que existen 22 empresas con un bajo rendimiento en comparación con las otras 38 .

A continuación, se presenta una tabla resumen de las frecuencias para esta variable, que ilustra de la información anterior.

\section{Tabla 1. Distribución de frecuencias del rendimiento}

\begin{tabular}{|c|c|c|c|c|c|c|}
\hline Variable & Clase & LI & LS & MC & FA & FR \\
\hline Rendimiento & 1 & 0,03 & 0,24 & 0,14 & 22 & 0,37 \\
Rendimiento & 2 & 0,24 & 0,44 & 0,34 & 17 & 0,28 \\
Rendimiento & 3 & 0,44 & 0,64 & 0,54 & 9 & 0,15 \\
Rendimiento & 4 & 0,64 & 0,85 & 0,75 & 10 & 0,17 \\
Rendimiento & 5 & 0,85 & 1,05 & 0,95 & 2 & 0,03 \\
\hline
\end{tabular}

El primer intervalo con mayor cantidad de empresas tiene una marca de clases de $14 \%$. Al comparar este promedio con la marca de clases del siguiente intervalo (clase 2) 34\%, el cual contiene a 17 empresas, se puede observar que este último es 2,4 veces mayor. El tercero, que representa un $15 \%$ de los datos, intervalo con una marca de clases de $54 \%$, es 3,8 veces superior al primero y 1,6 veces más alto que el promedio del segundo ítem. El cuarto intervalo contiene al $17 \%$ de los datos y tiene una marca de clase de $75 \%$, lo cual es 5,3 veces superior al primero, 2,2 veces superior que el segundo y 1,4 veces mayor que el tercer intervalo. Finalmente, el quinto ítem posee un promedio de $95 \%$ y representa el $2 \%$ de los datos; es 6,8 veces superior al primero, 2,8 veces superior al segundo, 1,75 veces mayor al tercero $y$ 1,3 veces más alto que el cuarto.

El siguiente gráfico presenta la distribución y la forma de los datos, en cuanto a las cinco clases señaladas anteriormente.

\section{Gráfico 1. Histograma variable rendlimiento}

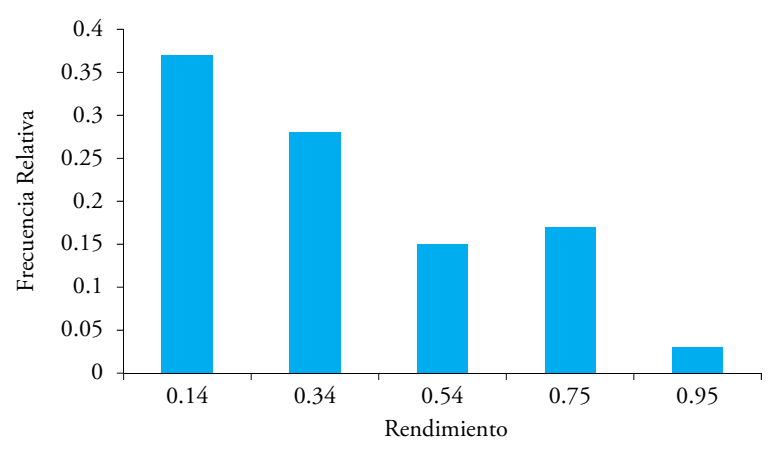

\subsubsection{Medidas de tendencia central}

El mínimo rendimiento obtenido por una organización de este ranking es de 3\%, mientras que, por otro lado, el máximo obtenido por una compañía fue de 105\%; esta última fue 35 veces superior al primero. La media de los datos es de $38 \%$, lo cual se encuentra dentro del segundo intervalo, y es 12,7 veces superior al mínimo y representa solo un $36,1 \%$ del rendimiento del valor máximo. La mediana (contenida en el segundo intervalo) de la muestra corresponde a 32\%; es decir, la mitad de los datos es igual o menos a este rendimiento. Al contrastar la mediana con la media, es visible alertar que esta última es mayor que la mitad de los datos, 1,19 veces mayor, lo que indica que la mitad de los datos está por debajo de la media. A su vez, la mediana representa un 33,7\% del rendimiento promedio del tercer intervalo, es decir, aquellas empresas con mayor retorno sobre la inversión del período en estudio.

A continuación, se expone una tabla que resume las medidas de tendencia central del rendimiento explicada anteriormente:

Tabla 2. Medidas descriptivas del rendimiento

\begin{tabular}{|c|c|c|c|c|c|}
\hline Variable & n & Media & Mín. & Máx. & Mediana \\
\hline Rendimiento & 60 & 0,38 & 0,03 & 1,05 & 0,32 \\
\hline
\end{tabular}




\subsubsection{Medidas de dispersión}

La desviación estándar de los datos corresponde a $25 \%$; en comparación con la media, se puede observar que es menor. Por lo tanto, a simple vista, no se puede asegurar alguna irregularidad en los datos. El cuartil 1 tiene un valor de $20 \%$; y el cuartil 3 , de $51 \%$, lo cual equivale a una diferencia intercuartil de $31 \%$.

En la siguiente tabla, se resumen las medidas de tendencia central y de dispersión, abordadas anteriormente.

\section{Tabla 3. Resumen de las medidas descriptivas y de dispersión del rendimiento}

\begin{tabular}{|c|c|c|c|c|c|c|c|c|}
\hline Variable & $\mathbf{n}$ & Media & D.E. Mín. Máx. Mediana & Q1 & Q3 \\
\hline Rendimiento & 60 & 0,38 & 0,25 & 0,03 & 1,05 & 0,32 & 0,2 & 0,51 \\
\hline
\end{tabular}

El dato extremo que se aprecia en el diagrama corresponde a una empresa que obtuvo para este período un rendimiento de $105 \%$.

Gráfico 2. Gráfico de caja del rendimiento

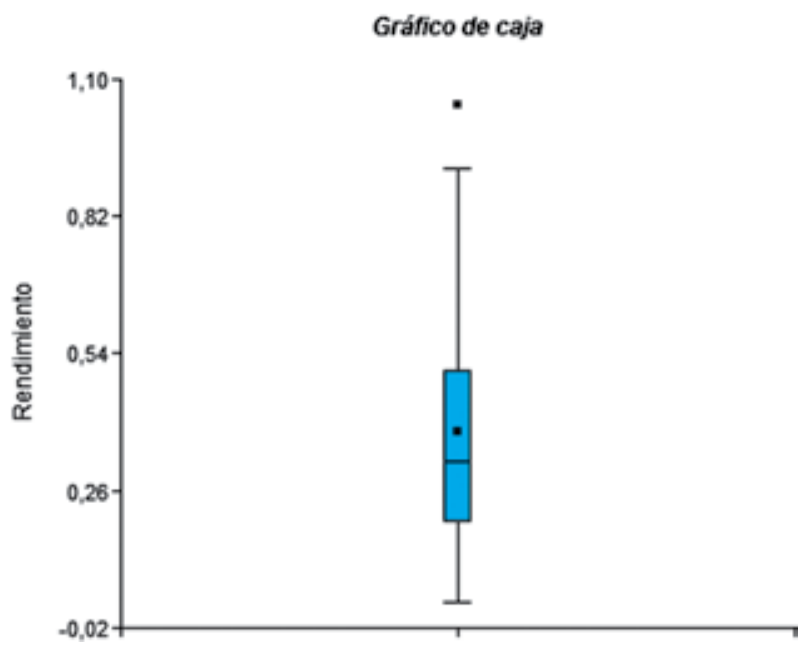

Luego de conocer las principales medidas estadísticas, se puede apreciar que existen grandes diferencias entre las empresas de este ranking. Considerando que el listado Fortune 500 agrupa a las compañías más grandes de Estados Unidos, se puede apreciar que, dentro del ranking, existen organizaciones con grandes retornos de inversión, a las que muchas otras entidades desearían alcanzar. Incluso, hay un caso en que el retorno supera el $100 \%$ de la inversión. Existe una alta diferencia entre el máximo y el mínimo: el primero es 35 veces superior al más bajo.

\subsection{Miembros del directorio}

\subsubsection{Medidas descriptivas}

Las medidas descriptivas para analizar la variable "miembros del directorio», que se refiere a la cantidad de miembros que tienen los directorios de las empresas del ranking Fortune 500 al año 2014, son el mínimo, el máximo, la media y la mediana. La cantidad mínima de miembros que tiene un directorio de la muestra del listado Fortune 500 del año 2014 es de 7 , mientras que, por otro lado, el máximo de miembros registrado por una junta es de 17 , lo cual supera en 2,4 veces al primero. La media de los datos es de 10,85 , muy cercana a 11 miembros, que es el número de miembros con mayor frecuencia dentro de los datos. La mediana es de 11 miembros, muy cercana al promedio de los datos, por lo que, teóricamente, es índice de una alta simetría en la distribución de los datos. En cuanto al promedio, este es 1,6 veces superior al mínimo de miembros encontrado dentro de los consejos de administración de las empresas y representa un $64,7 \%$ de la cantidad de miembros del máximo directorio encontrado.

\subsubsection{Medidas de dispersión}

La desviación estándar de la cantidad de miembros de los directorios de los miembros es de 1,92, muy cercana a la cantidad de 2 miembros. Al contrastar la media con la desviación estándar, no se puede 
establecer a simple vista una anomalía en la forma de los datos, debido a que esta última no supera el valor del promedio de los datos. Por otro lado, el cuartil 1 es de 9 miembros y el cuartil 3 es de 12, por lo que la diferencia intercuartil corresponde a 3 miembros; esta última es mayor a la desviación estándar de los datos.

A continuación, se presenta en conjunto las medidas descriptivas y de dispersión de la muestra de cantidad de miembros comentada anteriormente:

Tabla 4. Resumen de las medidas de descriptivas y de dispersión

\begin{tabular}{|c|c|c|c|c|c|c|c|c|}
\hline Variable & n & Media & D.E. Mín. Máx. Mediana Q1 & Q3 \\
\hline No de integrantes & 60 & 10,85 & 1,92 & 7 & 17 & 11 & 9 & 12 \\
\hline
\end{tabular}

\subsection{Variable independiente sexo}

\subsubsection{Distribución de frecuencias directores hombres}

El análisis de frecuencias se ha divido en 9 intervalos: comienza desde 4 hombres por directorio hasta llegar a 12 hombres en un directorio; ningún directorio tiene menos de 4 hombres en sus filas. El primer intervalo contempla a aquellas empresas con este número de hombres, en las que solo participa una compañía de la muestra, lo cual representa un $2 \%$ de los datos. En la clase siguiente, se incluye nuevamente una firma, que tiene 5 hombres dentro del directorio, y acumula un $2 \%$ de muestra. Para el intervalo 3, cuya marca de clases es de 6 hombres en una junta directiva, participan 6 organizaciones, que suman un $10 \%$ de los datos. La clase 4 incluye a 9 empresas, que tienen en su junta 97 directores hombres, lo cual constituye el 15\% de la muestra. El siguiente intervalo está formado por 9 empresas, que tienen 8 hombres en el consejo de administración; esto representa un 15\% del total. La clase 6 incorpora a 17 firmas que incluyen a 9 hombres dentro de la junta, con lo que ponderan un $28 \%$ de los datos; es la moda dentro de la muestra. El ítem 7 se refiere a compañías con 10 hombres en sus filas; dicho ítem está formado por 9 organizaciones, que alcanzan un $15 \%$ de los datos. La siguiente clase muestra a firmas con 11 hombres dentro del directorio e incluye a 6 compañías, que alcanzan un 10\% del total de empresas. Finalmente, se encuentra el ítem 9 con una marca de clase de 12 hombres por directorio, el cual está formado por 2 organizaciones que ponderan un $3 \%$ de la muestra.

A continuación, se presenta una tabla resumen de la frecuencia de los datos con respecto a la cantidad de hombres que se incluyen dentro de un directorio:

\section{Tabla 5. Distribución de frecuencias} de la cantidad de hombres

\begin{tabular}{|c|c|c|c|c|}
\hline Variable & Clase & MC & FA & FR \\
\hline Hombres & 1 & 4 & 1 & 0,02 \\
Hombres & 2 & 5 & 1 & 0,02 \\
Hombres & 3 & 6 & 6 & 0,1 \\
Hombres & 4 & 7 & 9 & 0,15 \\
Hombres & 5 & 8 & 9 & 0,15 \\
Hombres & 6 & 9 & 17 & 0,28 \\
Hombres & 7 & 10 & 9 & 0,15 \\
Hombres & 8 & 11 & 6 & 0,1 \\
Hombres & 9 & 12 & 2 & 0,03 \\
\hline
\end{tabular}

El siguiente histograma ilustra gráficamente la distribución de frecuencia de los datos. En su mayoría, los datos se encuentran agrupados en la cantidad de 7 a 10 , y representan un $73 \%$ de los datos, dentro de los cuales resalta el número 9 con $28 \%$. De acuerdo a lo anterior, las empresas de la muestra optan por incluir entre 7 a 10 hombres dentro del directorio. 
Gráfico 3. Histograma de la cantidad de hombres

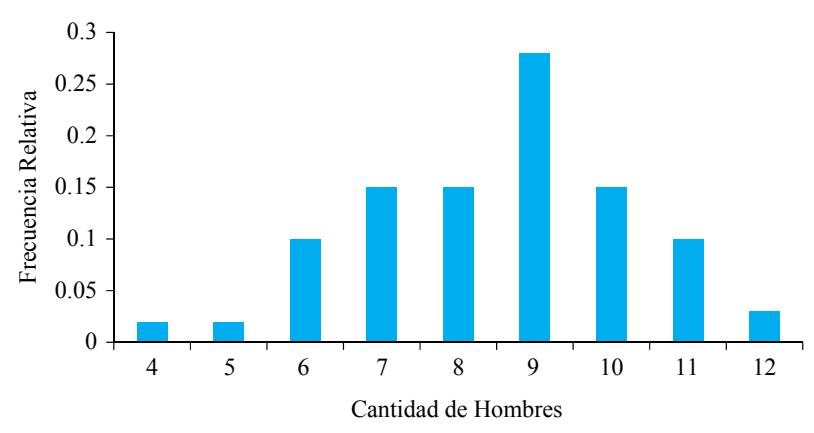

Medidas descriptivas: directores hombres

La cantidad mínima de miembros de un directorio corresponde a 4, mientras que el máximo corresponde a 12 hombres por directorio: por lo tanto, este último es 3 veces superior al primero. La media de los datos corresponde a 8,55 hombres por directorio, que, en aproximado, se refiere a 9, que, además, es el número de la moda de los datos. La mediana de los datos es de 9, por lo que, al contrastarla con los datos anteriores, se puede observar que tanto la media, la moda y la mediana son prácticamente el mismo número de hombres por directorio. Estas últimas 3 medidas representan alrededor de 2,25 veces mayor que el mínimo y el $75 \%$ del máximo de hombres en un directorio.

A continuación, se expone una tabla resumen de las medidas descriptivas de la cantidad de hombres encontradas dentro del directorio:

Tabla 6. Medidas descriptivas de la cantidad de hombres

\begin{tabular}{|c|c|c|c|c|c|}
\hline Variable & n & Media & Mín. & Máx. & Mediana \\
\hline Hombres & 60 & 8,55 & 4 & 12 & 9 \\
\hline
\end{tabular}

\section{Medidas de dispersión: directores hombres}

La desviación estándar de la cantidad de hombres del directorio corresponde a 1,74, más cercana a 2 que a 1 .
Al comparar la media con la desviación estándar, se puede observar que esta última no supera a la primera, por lo que a simple vista no se identifican señales de la presencia de peculiaridades en la distribución de los datos o la presencia de datos extremos o atípicos. Por otro lado, el cuartil uno es de 7 , mientras que el cuartil 2 corresponde a 10; por lo tanto, la diferencia intercurtil es de 3.

A continuación, se presenta una tabla que ilustra la información sobre las medidas descriptivas y las de dispersión de la cantidad de hombres que forman parte de los directorios de las empresas analizadas:

Tabla 7. Resumen de las variables descriptivas y de dispersión de la cantidad de hombres

\begin{tabular}{|c|c|c|c|c|c|c|c|c|}
\hline Variable & $\mathbf{n}$ & Media & D.E. & Mín. & Máx. & Mediana & Q1 & Q3 \\
\hline Hombres & 60 & 8,55 & 1,74 & 4 & 12 & 9 & 7 & 10 \\
\hline
\end{tabular}

El gráfico de caja revela que la forma de los datos no es simétrica, como se podría haber pensado debido a la proximidad de valores entre la media, la moda y la mediana.

Gráfico 4. Gráfico de caja de la cantidad de hombres

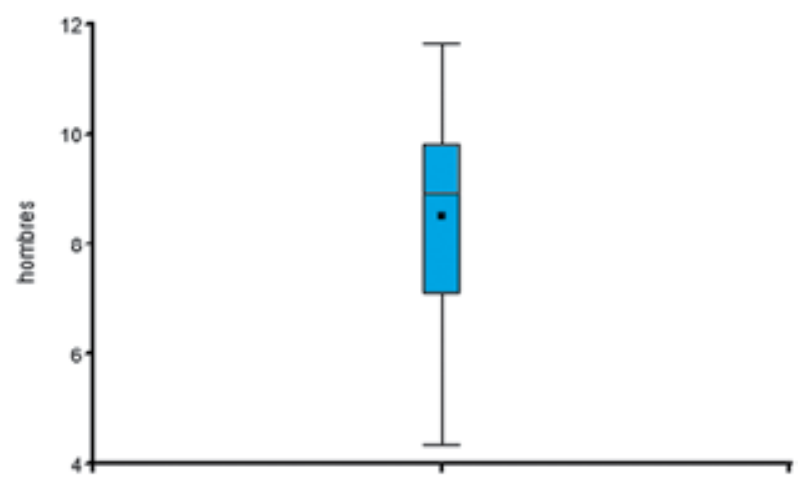

Luego del análisis descriptivo, se puede afirmar que la mayoría de los datos se concentra en 9 hombres por directorio; sin embargo, el rango que concentra más del $50 \%$ de los datos está entre 8 y 11 hombres por 
consejo de administración. Tanto la media, la moda y la mediana se aproximan en el valor de 9; no obstante, la distribución de los datos no es simétrica.

Si se compara esta variable con la cantidad de miembros, la que tenía una media de 11 , y la media de esta variable es 9, eso quiere decir, que en su mayoría los directorios tienen presencia masculina dentro de los participantes. Esto viene a ratificar lo estudiado en el marco teórico de este trabajo. De las 60 empresas estudiadas, existe solo un directorio que incluye la cifra de 4 hombres en la junta, mientras que existe una compañía incluye 12 hombres dentro del directorio. Como anteriormente se mencionó, la media de cantidad de miembros es 11 ; sin embargo, existen directorios con más integrantes. De estas, 6 firmas tienen 11 hombres y una 12, por lo que la presencia de hombres en el directorio, en algunos casos, iguala o supera al promedio de cantidad de miembros de los consejos de administración.

\subsubsection{Distribución de frecuencias: directores mujeres}

En análisis de frecuencias, se ha divido en seis intervalos. Existe dentro de la muestra 1 empresa que no incluye a ninguna mujer dentro del directorio, lo cual pondera un $2 \%$ de los datos y constituye la primera clase de distribución de frecuencias. El siguiente ítem incorpora a 17 compañías que incorporan 1 mujer dentro del directorio, lo cual representa un $28 \%$ de los datos. La clase 3 incluye a organizaciones con 2 mujeres en la junta directiva, $y$, al igual que el ítem anterior, acumula un 28\% de los datos. Cabe anotar que estas dos clases son las más repetidas dentro de la muestra. La siguiente en el listado de frecuencias es la clase 4, que incorpora a 16 empresas, que tienen en el consejo de administración 3 mujeres, lo cual pondera un 27\% del total de compañías. Luego, la clase 5 se refiere a las organizaciones que incluyen a 4 mujeres en el directorio y está constituido por 6 firmas, que repre- sentan el $10 \%$ de la muestra. Finalmente, el ítem 6 engloba a empresas con 5 mujeres en el directorio y está formado por 3 firmas, que acumulan un 5\% de los datos. El 98\% de las organizaciones incluye al menos 1 mujer dentro de la junta.

A continuación, se expone una tabla resumen de las frecuencias explicadas anteriormente sobre la participación de la mujer en los directorios de las empresas analizadas:

Tabla 8. Distribución de frecuencias de la cantidad de mujeres

\begin{tabular}{|l|c|c|c|c|}
\hline Variable & Clase & MC & FA & FR \\
\hline Mujeres & 1 & 0 & 1 & 0,02 \\
Mujeres & 2 & 1 & 17 & 0,28 \\
Mujeres & 3 & 2 & 17 & 0,28 \\
Mujeres & 4 & 3 & 16 & 0,27 \\
Mujeres & 5 & 4 & 6 & 0,1 \\
Mujeres & 6 & 5 & 3 & 0,05 \\
\hline
\end{tabular}

El histograma presentado a continuación muestra la distribución de la frecuencia abordada anteriormente. Los datos se encuentran agrupados en su mayoría en los ítems 2, 3 y 4 , lo que representa el $83 \%$ del total de empresas analizadas. De acuerdo a lo anterior, las compañías prefieren tener dentro de sus directorios entre 2 y 4 mujeres.

\section{Gráfico 5. Histograma de la cantidad de mujeres}

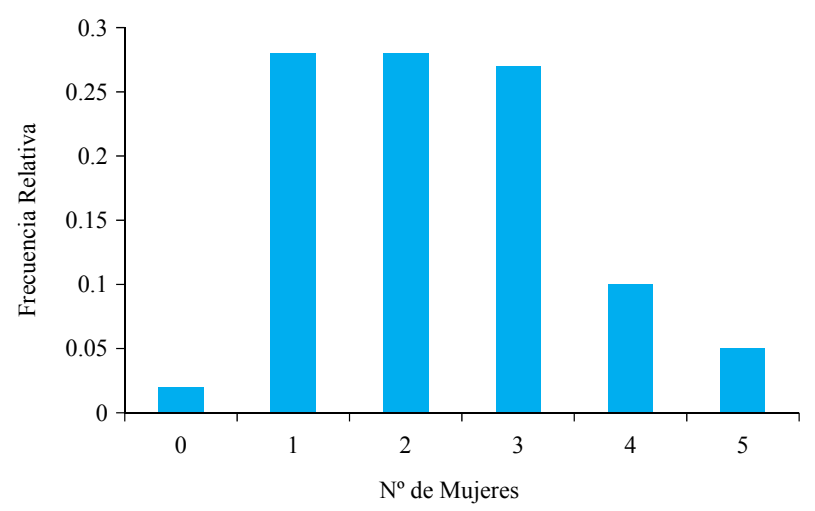




\section{Medidas descriptivas: directores mujeres}

En esta parte de la variable, la cantidad mínima incluida en un directorio es de cero; es decir, hay una empresa que no incluye en el directorio la participación femenina. Por otro lado, el máximo registrado en la muestra de empresas es de cinco mujeres en la junta directiva. La media de los datos corresponde a 2,30 , pero, como en este caso se refiere a personas, esta cantidad se acerca a 2 mujeres por directorio. La mediana de los datos se ubica en dos mujeres por junta directiva, muy parecida a la media de los datos. El 28\% de las firmas incluye a dos mujeres dentro del directorio.

A continuación, se presenta una tabla resumen de las medidas descriptivas de la cantidad de mujeres incluida en los directorios de las empresas:

Tabla 9. Medidas descriptivas de la cantidad de mujeres

\begin{tabular}{|c|c|c|c|c|c|}
\hline Variable & n & Media & Mín. & Máx. & Mediana \\
\hline Mujeres & 60 & 2,3 & 0 & 5 & 2 \\
\hline
\end{tabular}

\section{Medidas de dispersion: directores mujeres}

La desviación estándar de los datos corresponde a 1,18 , pero, como se trata de personas, está más cercana a 1 que a 2 mujeres. Al contrastar la media con la desviación estándar, no se puede predecir alguna particularidad en los datos o la presencia de datos atípicos, debido a que la medida de dispersión no supera al promedio. El valor para el cuartil 1 es de 1 , mientras que, para el cuartil 3, de 3. Por lo tanto, la diferencia intercuartil es de dos, muy parecida a la media de los datos.

A continuación, se muestra una tabla resumen de las medidas descriptivas y de dispersión de la cantidad de mujeres:

\section{Tabla 10. Medidas descriptivas y de dispersión} de la cantidad de mujeres

\begin{tabular}{|c|c|c|c|c|c|c|c|c|}
\hline Variable & n & Media & D.E. & Mín. & Máx. & Mediana & Q1 & Q3 \\
\hline Mujeres & 60 & 2,3 & 1,18 & 0 & 5 & 2 & 1 & 3 \\
\hline
\end{tabular}

El siguiente gráfico de caja ilustra la distribución de los datos, que no presenta datos atípicos y muestra una forma no simétrica, pero con gran proximidad de la media con la media.

Gráfico 6. Gráfico de caja de la cantidad de mujeres

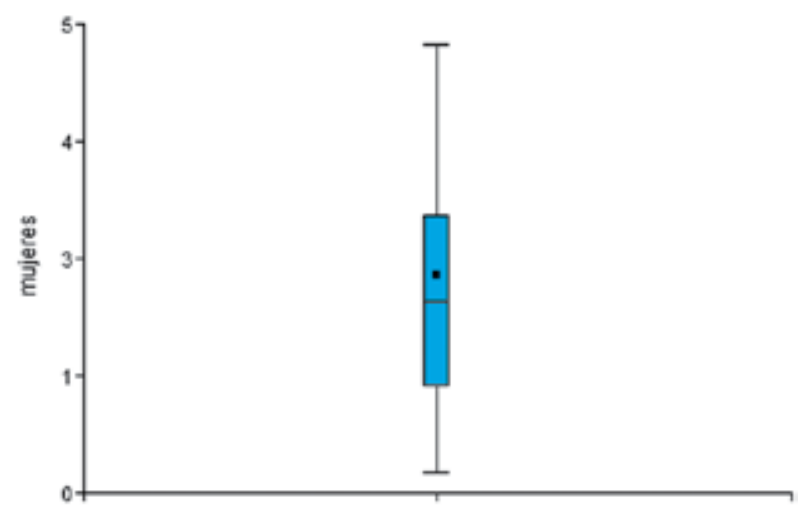

Después de un análisis descriptivo de la cantidad de mujeres presentes en el directorio, se puede observar que la mayoría de los datos se concentra en 2, 3 y 4, lo cual representa un $83 \%$ del total. Por otro lado, la media de los datos se encuentra cercana a 2 mujeres por directorio, cifra que, comparada con la media de la cantidad de miembros en las juntas equivalente a 11 personas, evidencia que la presencia femenina se manifiesta en un 18,18\% dentro del consejo de administración. Un 98\% de las empresas incluyen al menos una mujer dentro de la junta, por lo que se ratifica lo del marco teórico presentado sobre el hecho de que con el tiempo las compañías inevitablemente incorporaran mujeres en la alta dirección. La cifra máxima de participación femenina en un directorio se ubica en 5 
mujeres, solo en un 5\% de la muestra. Sin embargo, es un avance en la incorporación de la mujer en los consejos de administración de compañías tan importantes a nivel estadounidense y mundial.

\subsection{Aplicación Modelo Logit}

Para realizar este modelo de regresión cualitativa, se tomó respuesta de entre 1 y 0 , en que 1 es un rendimiento alto y 0 un rendimiento normal. Por otro lado, para convertir el sexo en una variable cuantitativa, es necesario convertirla a Dammy. Sobre esta base, 1 es hombre ( 512 observaciones) y 0 es mujer (138 observaciones). En cuanto a la observación de la variable rendimiento, se encontró 571 observaciones con rendimiento normal y 79 observaciones con rendimiento anormal.

La variable sexo, tiene un signo positivo. Esta variable es Dammy y, para el análisis, se tomó como base a la mujer por tener la denominación 0 . No obstante, esta variable no es estadísticamente significativa. Lo anterior responde a que el cálculo se realiza con un nivel de confianza del $90 \%$; por lo tanto, el valor de $\mathrm{z}$ es 1,645 y el estadístico resultante es 0,052, lo cual lo ubica dentro de la zona de confianza como muestra el siguiente gráfico:

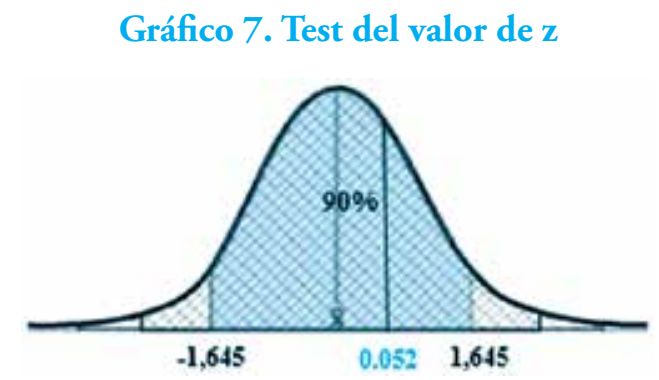

Esto indica que, para los datos incorporados en el análisis, el sexo no es preponderante para conseguir un rendimiento alto; por ende, el hecho de que los directivos sean hombres o mujeres no se relaciona con el rendimiento que obtenga la compañía.

\section{Discusión}

Existen grandes diferencias entre la cantidad de hombres y de mujeres incluidas en el directorio. El mínimo de hombres incluido en una junta corresponde a cuatro hombres, mientras que el mínimo número de mujeres es de cero en un consejo de administración. Por otro lado, el máximo número de hombres presente en las filas es de 12 y el de mujeres de 5; es decir, la cantidad de hombres es 2,4 veces superior.

La media para el caso de los hombres se ubica en 8,55 , que aproximadamente es de 9 miembros, mientras que la media para el caso de las mujeres es de 2,3, número que se aproxima más a 2 que a 3 , por lo que el número de hombre en promedio supera 4,5 veces la presencia de la mujer en las juntas directivas. Al contrastar estas medias con la media de la cantidad de miembros, se puede observar que los hombres representan $81,82 \%$ de la cantidad de miembros de un directorio y las mujeres $18,18 \%$.

A continuación, se presenta una tabla resumen con los datos de la cantidad de miembros de un directorio, cantidad de hombres y de mujeres:

Tabla 11. Resumen de medidas de la variable sexo

\begin{tabular}{|l|c|c|c|c|c|c|c|c|}
\hline \multicolumn{1}{|c|}{ Variable } & $\mathbf{n}$ & Media & D.E. & Mín. & Máx. & Mediana & Q1 & Q3 \\
\hline $\begin{array}{l}\text { No de inte- } \\
\text { grantes }\end{array}$ & 60 & 10,85 & 1,92 & 7 & 17 & 11 & 9 & 12 \\
\hline Hombres & 60 & 8,55 & 1,74 & 4 & 12 & 9 & 7 & 10 \\
\hline Mujeres & 60 & 2,3 & 1,18 & 0 & 5 & 2 & 1 & 3 \\
\hline
\end{tabular}

En el siguiente gráfico de caja, se observan las diferencias de la cantidad de hombres y de mujeres y la distribución de los datos: 
Gráfico 8. Gráfico de caja mujeres (amarillo) vs. hombres (verde)

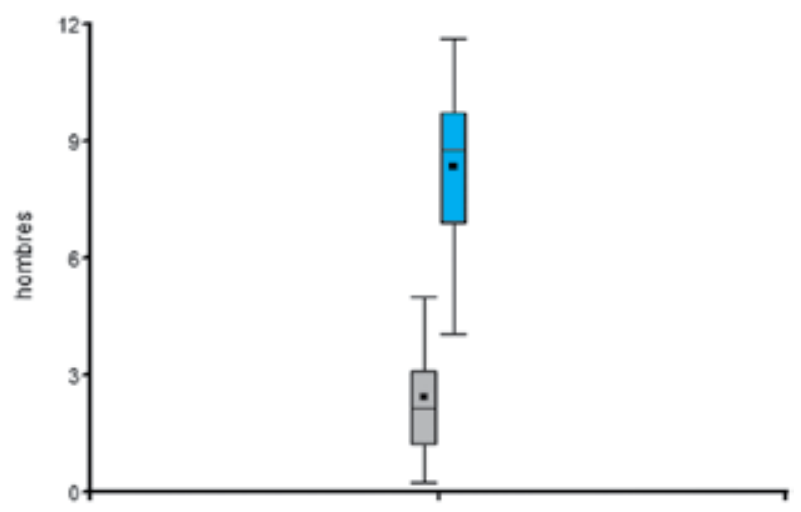

En Estados Unidos, para el año 2007, en un estudio del listado Fortune 500, se determinó que la mujer tenía una participación de $14,87 \%$ en los directorios (Johnson, Scholes y Whittington, 2006). Adams y Ferreira (2008) señalan que la inclusión de la mujer en los directorios está próxima a cambiar. En esta investigación, luego del análisis estadístico de 60 empresas del listado Fortune 500 del año 2014, el resultado de la participación de la mujer es de $18,18 \%$; por lo tanto, la inclusión femenina ha incrementado en 3,31 puntos porcentuales, lo que concuerda con lo señalado por estos autores (Adams \& Ferreira, 2008).

Sin embargo, luego de casi cinco años, la diferencia entre mujeres y hombres sigue siendo muy marcada. Estos autores no lograron probar una relación entre el rendimiento y la diversidad del directorio; en este trabajo, luego de observar las salidas del software, se puede ver que el estadístico obtenido no es estadísticamente significativo. El valor de $\mathrm{z}=0,052$ es un valor muy cercano a 0 , lo que deja entrever que el rendimiento es casi independiente de la variable sexo, por lo que el ser hombre o mujer no representa una probabilidad de tener mejor rendimiento. Por otro lado, de acuerdo a los datos ilustrados por Wheelen,
Hunder y Oliva (2007), un $80 \%$ de las empresas del listado Fortune 1000 incluía al menos a una mujer en el directorio. En esta investigación, se determina que en el listado Fortune 500, el 98\% de las empresas incluye al menos una mujer en el directorio.

\section{Conclusiones}

La participación de la mujer en los directorios de las empresas con mayores ventas de origen estadounidense es baja. Si bien con el pasar de los años ha aumentado, sigue siendo muy inferior a la presencia masculina, ya que, de once miembros en promedio que tiene una junta directiva, solo dos miembros en promedio son mujeres. Además de lo anterior, todavía hay empresas que no tienen presencia femenina entre los directores, lo que quiere decir que todavía la mujer no tiene la misma consideración que los hombres en altos cargos. Pese a que aún la situación es dispareja, hay autores que indican que esta situación está próxima a cambiar con el pasar de los años y la profesionalización de la mujer. En este trabajo, luego de un estudio estadístico de 60 empresas estadounidenses, se llegó a la conclusión de que el sexo no intervenía en el retorno sobre la inversión de una empresa, ya que, del total analizado, esta variable no fue estadísticamente significativa. La investigación presentada en este artículo es de un país catalogado como desarrollado, es decir, que tiene potencialidades distintas a países subdesarrollados o en vías de desarrollo, por lo que la pregunta que nace es "¿Qué sucede con estos países?», pues la literatura indica que la realidad no cambia e incluso la participación de la mujer es más baja. En Chile, por ejemplo, en un estudio realizado por el Servicio Nacional de la Mujer (2011), se concluyó que 31 de 155 directorios incluyen mujeres. De 31 directorios, 34 mujeres forman parte de ella, por lo que se podría afirmar que participa solo una mujer por directorio en promedio aproximadamente. De las 31 empresas, el $43 \%$ de las mujeres incluidas 
tiene relación familiar con los accionistas mayoritarios (Sernam, 2011). Lo anterior indica que, en la realidad, la incorporación de la mujer en los directorios de empresas chilenas es más baja aún y el índice de compañías que no incluye mujeres es de más del 50\%.

\section{Referencias}

Adams, Renée \& Daniel Ferreira (2004). Gender diversity in the boardroom. Estocolmo: Instituto Europeo de Gobierno Corporativo.

Adams, Renée \& Daniel Ferreira (2008). Women in the boardroom and their impact on governance and performance. Journal of Financial Economics, 94, 291309. https://doi.org/10.2139/ssrn.1107721

Bilimoria, Diana (2006). The relationship between women corporate directors and women corporate officers. Journal of Managerial, 18(1), 47-61.

Bilimoria, Diana \& Sandy Piderit (1994). Board Committee Membership Effects of sex-based bias. Academy of Management Journal, 37(6), 1453-1477. https://doi. org/10.2307/256795

Burgess, Zena \& Phyllis Tharenou (2002). Women Board Directors: Characteristics of the Few. Journal of Business Ethics, 37, 39-49. https://doi. org/10.1023/A:1014726001155

Caprino, Kathy (2013). The top 6 Reasons Women Are Not Leading In Corporate America as we need them to. Forbes, Consulta: 10 de setiembre de 2015 http:// www.forbes.com/sites/kathycaprino/2013/02/12/ the-top-6-reasons-women-are-not-leading-in-corporate-america-as-we-need-them-to/\#5f475ba16d6d

Carter, D. A., B. J. Simkins \& W. G. Simpson (2002). Corporate governance, board diversity and firm value. Oklahoma State University, working paper. Disponível em: <http://ssrn.com/abstract=304499

Catalyst(1995).Equityinbusinessleadership. Catalyst, 15 deabril. Consulta: 15 de noviembre de 2014. http://www.catalyst. org/knowledge/ceo-view-women-corporate-boards

Erhardt, Niclas, James Werbel \& Charles Shrader (2003). Board of director diversity and firm financial performance.
Corporate Governance: An International Review, 11(2), 102-111. https://doi.org/10.1111/1467-8683.00011

Ernst \& Young (2013). EY. 30 de setiembre. Consulta: 11 de agosto de 2014. http://www.ey.com/Publication/ vwLUAssets/EY-Diversity-drives-diversity/\$FILE/EYDiversity-drives-diversity.pdf

Fortune (2014). Fortune. Consulta: 25 de julio de 2014. http://fortune.com/fortune500/

Jara, Natalia (2014). Gobierno corporativo: una revisión. Santiago: Universidad de Chile.

Johnson, Garry, Kevan Scholes \& Richard Whittington (2006). Dirección estratégica. Madrid: Pearson.

Kuczynski, Sherry (1999). If diversity, then higher profits. HR Magazine, 44(13), 66-71.

Oakley, Judith (2000). Gender-based barriers to senior management positions: Understanding the scarcity of female CEOs. Journal of Business Ethics, 27(4), 321334. https://doi.org/10.1023/A:1006226129868

Sernam (2011). Servicio Nacional de la Mujer. Consulta: 23 de septiembre de 2014. http://estudios.sernam.cl/ ? $\mathrm{m}=\mathrm{e} \& \mathrm{i}=181$

Tacheva, Sabina y Morten Huse (2006). Women directors and board task performance: Mediating and moderating effects of board working style. Ponencia presentada en EURAM meeting 2006. Oslo.

Torchia, Mariateresa, Andrea Calabro \& Morten Huse (2011). Women directors on corporate boards: From tokenism to critical mass. Journal of Business Ethics, 102(2), pp. 299317. https://doi.org/10.1007/s10551-011-0815-z

Wheelen, Thomas, J. David Hunger \& Ismael Oliva (2007). Administración estratégica y política de negocio. México: Pearson.

Williams, Robert (2003). Women on corporate boards of directors and their influence on corporate philanthropy. Journal of Business Ethics, 42(1), 1-10. https://doi.org/10.1023/A:1021626024014

Fecha de recepción: 23 de marzo del 2016 Fecha de aceptación: 18 de octubre del 2016 Correspondencia: barbarareyes289@gmail.com alex@ubiobio.cl 